\title{
Diagnostic one-stop-shop in suspected coronary artery disease gets closer. Coronary computed tomography angiography based fractional flow reserve
}

\author{
Mariusz Kruk \\ Coronary Artery and Structural Heart Diseases Department, Institute of Cardiology, Warsaw, Poland
}

Adv Interv Cardiol 2016; 12, 2 (44): 93-95

DOI: 10.5114/aic.2016.59357

Current diagnostics of suspected coronary artery disease (CAD) significantly relies on coronary angiography. The traditional, invasive diagnostics is recommended in patients with high, but non-invasive coronary computed tomography angiography (CTA) in patients with intermediate pre-test probability of significant CAD [1]. Basically, the angiography is designed to reflect the anatomical status of the coronary artery lumen, especially concentrating on the presence and the degree of stenosis. Both anatomic methods of examination suffer from poor correlation with outcomes of functional testing for ischemia. Notably, invasive angiography is not superior in this respect to coronary CTA [2]. There is a consensus, reflected in the ESC guidelines, that stenoses above $90 \%$ are significant, i.e. allegedly cause ischemia, and those less than $50 \%$ are harmless. What lies between these thresholds, however, is called intermediate stenosis, and usually requires further functional evaluation prior to the potential intervention.

Given the indications for coronary CTA, relatively frequent diagnosis of intermediate coronary stenosis (50$90 \%$ ) in a patient with intermediate probability of CAD, borderline symptoms or an equivocal functional test is particularly perplexing. On the other hand, patients with indications for invasive angiography should have typical symptoms, or confirmed ischemia, which limits the number of functionally ambiguous stenoses in this group. The lack of conclusive diagnosis in a significant number of patients with intermediate stenosis poses a serious limitation of coronary CTA, as it leads to further functional testing. Therefore, any additional information derived from the CTA datasets, which would confidently limit the grey zone of borderline stenoses, is desirable.

A gold standard for management of intermediate coronary stenoses in the catheterization laboratory com- prises measurement of fractional flow reserve (FFR). Its appeal is based on diagnostic straightforwardness and perceived conceptual simplicity, providing a quick and concrete solution to the problem. The unequivocal threshold of $\leq 0.80$ confirmed in randomized trials and endorsed by guidelines provides assurance to the operators. The first mention about potential application of the FFR concept to coronary CTA datasets can be traced back to the beginning of the twenty-first century. The main methodological barriers for simulation of the trans-lesional gradient was a difficulty in establishing how much blood flowed in and out of the specific branches of coronary arteries (so-called boundary conditions for the flow models), the requirement for huge amounts of computing power, and borderline resolution of CTA, especially in the presence of calcifications [3]. According to basic equations of fluid mechanics, the pressure loss across the stenosis is very sensitive to minimum lumen area (quadratic function); therefore, even small inaccuracies in coronary lumen delineation could lead to significant errors. Continuous hardware and software technological development led to improved coronary CTA resolution $(0.3-0.5 \mathrm{~mm})$, diminishing the impact of calcifications, and increasing available computational power. The startup company HeartFlow Inc. was the first to successfully marry the advanced imaging and computational fluid dynamics technologies, and in 2010 presented the first results of CTA-based FFR calculations performed in a clinical environment. The accuracy of CTA-FFR, according to the DISCOVER-FLOW study, was decent at $84 \%$, and significantly better than the criterion based on the 50\% angiographic stenosis threshold (59\%) [4]. The next study (Determination of Fractional Flow Reserve by Anatomic Computed Tomographic Angiography - DeFACTO) showed even worse reclassification power of CTA-FFR

\section{Corresponding author:}

Mariusz Kruk MD, Coronary Artery and Structural Heart Diseases Department, Institute of Cardiology, 42 Alpejska St, 04-628 Warsaw, Poland, phone: +48 601145 011, e-mail: mariuszkruk2000@yahoo.com

Received: 29.02.2016, accepted: 29.02.2016. 
than the previous study at $73 \%$; importantly, only $9 \%$ more patients were reclassified correctly as compared to the angiographic criterion of 50\% stenosis [5]. Parallel to improved calculation algorithms, the diagnostic accuracy of the model improved in the NXT trial, reaching $86 \%$ [3]. However, the relatively high accuracy did not translate into much improved reclassification over the angiographic criterion of $50 \%$ ( $21 \%$ of patients), suggesting that at least partially the high accuracy was secondary to extreme (either severe or obviously non-significant) angiographic characteristics of the stenoses. In the meantime, the second CTA-based prototype FFR system was reported (Siemens A.G.); its accuracy was comparable to the competitor's method [6]. The overall positive results of the studies, and the bulk of evidence, led to FDA approval of the Heartflow Inc. method, allowing its clinical use. The current paper of Dai et al. reports the outcomes of another, in-house engineered CTA-based FFR system, with outstanding sensitivity and specificity at $85 \%$ and 93\% respectively, numerically better than that of either Heartflow's or Siemens'. The reported high accuracy must be treated cautiously due to methodological inferiority including single-center design, moderate number of patients, non-consecutive cohort, single vessel disease and lack of validation on an independent cohort [7]. It must be noted that despite a similar basic concept, each of the CTA-FFR simulations is based on individual, proprietary algorithms, comprising different methods and therefore requiring independent validation.

Along with FDA approval of the first CTA-FFR system, it may seem that it is ripe for use in the clinical environment. However, there are several issues to be addressed before the method is widely implemented. First of all, the practical elegance of the reference, invasive FFR itself is misleading, as it has serious limitations. The measurement repeatability of a single FFR result is $>95 \%$ only outside the gray zone of $0.75-0.85$, and closer to its cutoff the certainty falls, to less than $80 \%$ within 0.77 to 0.83 , and a nadir of $50 \%$ around the 0.8 threshold [8]. A further dent in the value of invasive FFR is inflicted by outcomes of the RIPCORD (Does Routine Pressure Wire Assessment Influence Management Strategy at Coronary Angiography for Diagnosis of Chest Pain) study, where even $13 \%$ of mild $(0-30 \%)$ stenoses had FFR $\leq 0.8$, and the same $33 \%$ proportion of significant FFR was reported for both $31-50 \%$ and $51-70 \%$ stenoses, and for only $53 \%$ of stenoses above $70 \%$ [9].

Current literature referring to the simulated CTA-FFR, including the post-marketing PLATFORM study of Heartflow Inc., seems to ignore the fact that CTA-FFR is not homologous to invasive FFR, and postulates use of the $\leq 0.80$ threshold to make clinical decisions based on the CTA-FFR [3-5]. However, given limits of agreement at around $20 \%$, and an average accuracy of the simulated FFR at around $80 \%$ respective to the reference method with $80 \%$ repeatability itself in the range of $0.75-0.85$, the postulated clear-cut threshold seems to be a misconception, as it may lead to erroneous qualifications in nearly $50 \%$ of borderline FFR cases. A partial solution to the problem may be optimization of the diagnostic accuracy parameters of the specific CTA-FFR, by defining the individual cut-off for each method, as proposed by Dai et al. in Advances in Interventional Cardiology [7]. However, given the simulated FFR relatively wide limits of agreement, the relevant question is rather: "What proportion of patients can be confidently diagnosed with the specific CTA-FFR?" Our previous analysis of prototype CTA-FFR showed that the method was able to confidently (both positive and negative predictive values $\geq 90 \%$ ) diagnose around half of the lesions displaying intermediate stenosis (50-90\% on CTA), using the threshold of either $>0.87$ or $<0.74$ for respectively nonsignificant or significant stenosis [10]. These results seem to discourage use of the single thresholds for the clinical application of CTA-FFR, unless both negative and positive predictive values are sufficiently high for the single point. Although this approach proved inconclusive for around $50 \%$ of patients with intermediate stenosis on CTA, it brought a relatively confident diagnosis for the remainder.

CTA-based FFR currently remains a diagnostic method under development, but its huge potential is already emerging. Although it is unknown how it will ultimately be applied in the clinical environment, even under current technology it may provide a diagnostic "one-stopshop" for around $50 \%$ of patients, who currently are referred for further testing due to intermediate stenosis on CTA. Future developments in both image quality and computational fluid dynamics algorithms will likely improve CTA-FFR accuracy, with fewer patients left in the "waiting room" of the gray diagnostic zone.

\section{Conflict of interest}

The author declares no conflict of interest.

\section{References}

1. Task Force Members, Montalescot G, Sechtem U, Achenbach S, et al. 2013 ESC guidelines on the management of stable coronary artery disease: the Task Force on the management of stable coronary artery disease of the European Society of Cardiology. Eur Heart J 2013; 34: 2949-3003.

2. Budoff MJ, Nakazato R, Mancini GB, et al. CT angiography for the prediction of hemodynamic significance in intermediate and severe lesions: head-to-head comparison with quantitative coronary angiography using fractional flow reserve as the reference standard. JACC Cardiovasc Imaging 2016 Feb 10. pii: S1936-878X(15)00973-0.

3. Nørgaard BL, Leipsic J, Gaur S, et al.; NXT Trial Study Group. Diagnostic performance of noninvasive fractional flow reserve derived from coronary computed tomography angiography in suspecteds coronary artery disease: the NXT trial (Analysis of 
Coronary Blood Flow Using CT Angiography: Next Steps). J Am Coll Cardiol 2014; 63: 1145-55.

4. Koo BK, Erglis A, Doh JH, et al. Diagnosis of ischemia-causing coronary stenoses by noninvasive fractional flow reserve computed from coronary computed tomographic angiograms. Results from the prospective multicenter DISCOVER-FLOW (Diagnosis of Ischemia-Causing Stenoses Obtained Via Noninvasive Fractional Flow Reserve) study. J Am Coll Cardiol 2011; 58: 1989-97.

5. Min JK, Leipsic J, Pencina MJ, et al. Diagnostic accuracy of fractional flow reserve from anatomic CT angiography. JAMA 2012; 308: 1237-45.

6. Renker M, Schoepf UJ, Wang R, et al. Comparison of diagnostic value of a novel noninvasive coronary computed tomography angiography method versus standard coronary angiography for assessing fractional flow reserve. Am J Cardiol 2014; 114: 1303-8.

7. Dai N, Lv HJ, Xiang YF, et al. Three-dimensional modeling and numerical analysis of fractional flow reserve in human coronary arteries. Adv Interv Cardiol 2016; 12: 25-31.

8. Petraco R, Sen S, Nijjer S, et al. Fractional flow reserve-guided revascularization: practical implications of a diagnostic gray zone and measurement variability on clinical decisions. JACC Cardiovasc Interv 2013; 6: 222-5.

9. Curzen N, Rana O, Nicholas Z, et al. Does routine pressure wire assessment influence management strategy at coronary angiography for diagnosis of chest pain? The RIPCORD study. Circ Cardiovasc Interv 2014; 7: 248-55.

10. Kruk M, Wardziak Ł, Demkow M, et al. Workstation-based calculation of CTA-based FFR for intermediate stenosis. JACC Cardiovasc Imaging 2016, doi: 10.1016/j.jcmg.2015.09.019. 